\title{
Three-point boundary value problems for nonlinear second-order impulsive $q$-difference equations
}

\author{
Jessada Tariboon ${ }^{1 *}$ and Sotiris K Ntouyas ${ }^{2}$
}

"Correspondence:

jessadat@kmutnb.ac.th

${ }^{1}$ Department of Mathematics,

Faculty of Applied Science, King Mongkut's University of Technology

North Bangkok, Bangkok, Thailand

Full list of author information is

available at the end of the article

\begin{abstract}
The quantum calculus on finite intervals was studied recently by the authors in Adv. Differ. Equ. 2013:282, 2013, where the concepts of $q_{k}$-derivative and $q_{k}$-integral of a function $f: J_{k}:=\left[t_{k}, t_{k+1}\right] \rightarrow \mathbb{R}$ have been introduced. In this paper, we prove existence and uniqueness results for nonlinear second-order impulsive $q_{k}$-difference three-point boundary value problems, by using Banach's contraction mapping principle and Krasnoselskii's fixed-point theorem.
\end{abstract}

MSC: 26A33; 39A13; 34A37

Keywords: $q_{k}$-derivative; $q_{k}$-integral; impulsive $q_{k}$-difference equation; existence; uniqueness; three-point boundary conditions; fixed-point theorems

\section{Introduction}

In this article, we investigate the nonlinear second-order impulsive $q_{k}$-difference equation with three-point boundary conditions

$$
\left\{\begin{array}{l}
D_{q_{k}}^{2} x(t)=f(t, x(t)), \quad t \in J:=[0, T], t \neq t_{k}, \\
\Delta x\left(t_{k}\right)=I_{k}\left(x\left(t_{k}\right)\right), \quad k=1,2, \ldots, m, \\
D_{q_{k}} x\left(t_{k}^{+}\right)-D_{q_{k-1}} x\left(t_{k}\right)=I_{k}^{*}\left(x\left(t_{k}\right)\right), \quad k=1,2, \ldots, m, \\
x(0)=0, \quad x(T)=x(\eta),
\end{array}\right.
$$

where $0=t_{0}<t_{1}<t_{2}<\cdots<t_{k}<\cdots<t_{m}<t_{m+1}=T, f: J \times \mathbb{R} \rightarrow \mathbb{R}$ is a continuous function, $I_{k}, I_{k}^{*} \in C(\mathbb{R}, \mathbb{R}), \Delta x\left(t_{k}\right)=x\left(t_{k}^{+}\right)-x\left(t_{k}\right)$ for $k=1,2, \ldots, m, x\left(t_{k}^{+}\right)=\lim _{h \rightarrow 0} x\left(t_{k}+h\right), \eta \in\left(t_{j}, t_{j+1}\right)$ a constant for some $j \in\{0,1,2, \ldots, m\}$ and $0<q_{k}<1$ for $k=0,1,2, \ldots, m$.

The theory of quantum calculus on finite intervals was developed recently by the authors in [1]. In [1] the concepts of $q_{k}$-derivative and $q_{k}$-integral of a function $f: J_{k}:=\left[t_{k}, t_{k+1}\right] \rightarrow$ $\mathbb{R}$, are defined and their basic properties proved. As applications, existence and uniqueness results for initial value problems for first- and second-order impulsive $q_{k}$-difference equations are proved.

The book by Kac and Cheung [2] covers many of the fundamental aspects of the quantum calculus. In recent years, the topic of $q$-calculus has attracted the attention of several researchers and a variety of new results can be found in the papers [3-15] and the references cited therein.

C2014 Tariboon and Ntouyas; licensee Springer. This is an Open Access article distributed under the terms of the Creative Commons Attribution License (http://creativecommons.org/licenses/by/2.0), which permits unrestricted use, distribution, and reproduction in any medium, provided the original work is properly cited. 
Impulsive differential equations, that is, differential equations involving an impulse effect, appear as a natural description of observed evolution phenomena of several realworld problems. For some monographs on impulsive differential equations we refer to [16-18].

In the present paper we prove existence and uniqueness results for the impulsive boundary value problem (1.1) by using Banach's contraction mapping principle and Krasnoselskii's fixed-point theorem. The rest of this paper is organized as follows: In Section 2 we present the notions of $q_{k}$-derivative and $q_{k}$-integral on finite intervals and collect their properties. The main results are proved in Section 3, while examples illustrating the results are presented in Section 4.

\section{Preliminaries}

In this section we present the notions of $q_{k}$-derivative and $q_{k}$-integral on finite intervals. For a fixed $k \in \mathbb{N} \cup\{0\}$ let $J_{k}:=\left[t_{k}, t_{k+1}\right] \subset \mathbb{R}$ be an interval and $0<q_{k}<1$ be a constant. We define $q_{k}$-derivative of a function $f: J_{k} \rightarrow \mathbb{R}$ at a point $t \in J_{k}$ as follows.

Definition 2.1 Assume $f: J_{k} \rightarrow \mathbb{R}$ is a continuous function and let $t \in J_{k}$. Then the expression

$$
D_{q_{k}} f(t)=\frac{f(t)-f\left(q_{k} t+\left(1-q_{k}\right) t_{k}\right)}{\left(1-q_{k}\right)\left(t-t_{k}\right)}, \quad t \neq t_{k}, \quad D_{q_{k}} f\left(t_{k}\right)=\lim _{t \rightarrow t_{k}} D_{q_{k}} f(t)
$$

is called the $q_{k}$-derivative of function $f$ at $t$.

We say that $f$ is $q_{k}$-differentiable on $J_{k}$ provided $D_{q_{k}} f(t)$ exists for all $t \in J_{k}$. Note that if $t_{k}=0$ and $q_{k}=q$ in (2.1), then $D_{q_{k}} f=D_{q} f$, where $D_{q}$ is the well-known $q$-derivative of the function $f(t)$ defined by

$$
D_{q} f(t)=\frac{f(t)-f(q t)}{(1-q) t}
$$

In addition, we should define the higher $q_{k}$-derivative of functions.

Definition 2.2 Let $f: J_{k} \rightarrow \mathbb{R}$ is a continuous function, we call the second-order $q_{k}$ derivative $D_{q_{k}}^{2} f$ provided $D_{q_{k}} f$ is $q_{k}$-differentiable on $J_{k}$ with $D_{q_{k}}^{2} f=D_{q_{k}}\left(D_{q_{k}} f\right): J_{k} \rightarrow \mathbb{R}$. Similarly, we define the higher-order $q_{k}$-derivative $D_{q_{k}}^{n}: J_{k} \rightarrow \mathbb{R}$.

The properties of the $q_{k}$-derivative are summarized in the following theorem.

Theorem 2.3 Assume $f, g: J_{k} \rightarrow \mathbb{R}$ are $q_{k}$-differentiable on $J_{k}$. Then:

(i) The sum $f+g: J_{k} \rightarrow \mathbb{R}$ is $q_{k}$-differentiable on $J_{k}$ with

$$
D_{q_{k}}(f(t)+g(t))=D_{q_{k}} f(t)+D_{q_{k}} g(t) .
$$

(ii) For any constant $\alpha, \alpha f: J_{k} \rightarrow \mathbb{R}$ is $q_{k}$-differentiable on $J_{k}$ with

$$
D_{q_{k}}(\alpha f)(t)=\alpha D_{q_{k}} f(t) .
$$


(iii) The product $f g: J_{k} \rightarrow \mathbb{R}$ is $q_{k}$-differentiable on $J_{k}$ with

$$
\begin{aligned}
D_{q_{k}}(f g)(t) & =f(t) D_{q_{k}} g(t)+g\left(q_{k} t+\left(1-q_{k}\right) t_{k}\right) D_{q_{k}} f(t) \\
& =g(t) D_{q_{k}} f(t)+f\left(q_{k} t+\left(1-q_{k}\right) t_{k}\right) D_{q_{k}} g(t) .
\end{aligned}
$$

(iv) If $g(t) g\left(q_{k} t+\left(1-q_{k}\right) t_{k}\right) \neq 0$, then $\frac{f}{g}$ is $q_{k}$-differentiable on $J_{k}$ with

$$
D_{q_{k}}\left(\frac{f}{g}\right)(t)=\frac{g(t) D_{q_{k}} f(t)-f(t) D_{q_{k}} g(t)}{g(t) g\left(q_{k} t+\left(1-q_{k}\right) t_{k}\right)} .
$$

Definition 2.4 Assume $f: J_{k} \rightarrow \mathbb{R}$ is a continuous function. Then the $q_{k}$-integral is defined by

$$
\int_{t_{k}}^{t} f(s) d_{q_{k}} s=\left(1-q_{k}\right)\left(t-t_{k}\right) \sum_{n=0}^{\infty} q_{k}^{n} f\left(q_{k}^{n} t+\left(1-q_{k}^{n}\right) t_{k}\right),
$$

for $t \in J_{k}$. Moreover, if $a \in\left(t_{k}, t\right)$ then the definite $q_{k}$-integral is defined by

$$
\begin{aligned}
\int_{a}^{t} f(s) d_{q_{k}} s= & \int_{t_{k}}^{t} f(s) d_{q_{k}} s-\int_{t_{k}}^{a} f(s) d_{q_{k}} s \\
= & \left(1-q_{k}\right)\left(t-t_{k}\right) \sum_{n=0}^{\infty} q_{k}^{n} f\left(q_{k}^{n} t+\left(1-q_{k}^{n}\right) t_{k}\right) \\
& -\left(1-q_{k}\right)\left(a-t_{k}\right) \sum_{n=0}^{\infty} q_{k}^{n} f\left(q_{k}^{n} a+\left(1-q_{k}^{n}\right) t_{k}\right) .
\end{aligned}
$$

Note that if $t_{k}=0$ and $q_{k}=q$, then (2.3) reduces to the $q$-integral of a function $f(t)$, defined by $\int_{0}^{t} f(s) d_{q} s=(1-q) t \sum_{n=0}^{\infty} q^{n} f\left(q^{n} t\right)$ for $t \in[0, \infty)$.

Theorem 2.5 For $t \in J_{k}$, the following formulas hold:

(i) $D_{q_{k}} \int_{t_{k}}^{t} f(s) d_{q_{k}} s=f(t)$;

(ii) $\int_{t_{k}}^{t} D_{q_{k}} f(s) d_{q_{k}} s=f(t)$;

(iii) $\int_{a}^{t} D_{q_{k}} f(s) d_{q_{k}} s=f(t)-f(a)$ for $a \in\left(t_{k}, t\right)$.

\section{Main results}

Let $J=[0, T], J_{0}=\left[t_{0}, t_{1}\right], J_{k}=\left(t_{k}, t_{k+1}\right]$ for $k=1,2, \ldots, m$. Let $P C(J, \mathbb{R})=\{x: J \rightarrow \mathbb{R}:$ $x(t)$ is continuous everywhere except for some $t_{k}$ at which $x\left(t_{k}^{+}\right)$and $x\left(t_{k}^{-}\right)$exist and $x\left(t_{k}^{-}\right)=$ $\left.x\left(t_{k}\right), k=1,2, \ldots, m\right\} . P C(J, \mathbb{R})$ is a Banach space with the norm $\|x\|_{P C}=\sup \{|x(t)| ; t \in J\}$.

Lemma 3.1 The unique solution of problem (1.1) is given by

$$
\begin{aligned}
x(t)= & -t \sum_{k=1}^{j}\left(\int_{t_{k-1}}^{t_{k}} f(s, x(s)) d_{q_{k-1}} s+I_{k}^{*}\left(x\left(t_{k}\right)\right)\right) \\
& -\frac{t}{T-\eta} \sum_{k=j+1}^{m}\left(\int_{t_{k-1}}^{t_{k}} \int_{t_{k-1}}^{s} f(\sigma, x(\sigma)) d_{q_{k-1}} \sigma d_{q_{k-1}} s+I_{k}\left(x\left(t_{k}\right)\right)\right)
\end{aligned}
$$




$$
\begin{aligned}
& -\frac{t}{T-\eta} \sum_{k=j+1}^{m}\left(\int_{t_{k-1}}^{t_{k}} f(s, x(s)) d_{q_{k-1}} s+I_{k}^{*}\left(x\left(t_{k}\right)\right)\right)\left(T-t_{k}\right) \\
& +\frac{t}{T-\eta} \int_{t_{j}}^{\eta} \int_{t_{j}}^{s} f(\sigma, x(\sigma)) d_{q_{j}} \sigma d_{q_{j}} s \\
& -\frac{t}{T-\eta} \int_{t_{m}}^{T} \int_{t_{m}}^{s} f(\sigma, x(\sigma)) d_{q_{m}} \sigma d_{q_{m}} s \\
& +\sum_{0<t_{k}<t}\left(\int_{t_{k-1}}^{t_{k}} \int_{t_{k-1}}^{s} f(\sigma, x(\sigma)) d_{q_{k-1}} \sigma d_{q_{k-1}} s+I_{k}\left(x\left(t_{k}\right)\right)\right) \\
& +\sum_{0<t_{k}<t}\left(\int_{t_{k-1}}^{t_{k}} f(s, x(s)) d_{q_{k-1}} s+I_{k}^{*}\left(x\left(t_{k}\right)\right)\right)\left(t-t_{k}\right) \\
& +\int_{t_{k}}^{t} \int_{t_{k}}^{s} f(\sigma, x(\sigma)) d_{q_{k}} \sigma d_{q_{k}} s,
\end{aligned}
$$

with $\sum_{0<0}(\cdot)=0$.

Proof For $t \in J_{0}$, taking the $q_{0}$-integral for the first equation of (1.1), we get

$$
D_{q_{0}} x(t)=D_{q_{0}} x(0)+\int_{0}^{t} f(s, x(s)) d_{q_{0}} s
$$

which yields

$$
D_{q_{0}} x\left(t_{1}\right)=D_{q_{0}} x(0)+\int_{0}^{t_{1}} f(s, x(s)) d_{q_{0}} s .
$$

For $t \in J_{0}$ we obtain by $q_{0}$-integrating (3.2),

$$
\begin{aligned}
x(t) & =x(0)+D_{q_{0}} x(0) t+\int_{0}^{t} \int_{0}^{s} f(\sigma, x(\sigma)) d_{q_{0}} \sigma d_{q_{0}} s \\
& :=A+B t+\int_{0}^{t} \int_{0}^{s} f(\sigma, x(\sigma)) d_{q_{0}} \sigma d_{q_{0}} s \quad\left(x(0)=A, D_{q_{0}} x(0)=B\right) .
\end{aligned}
$$

In particular, for $t=t_{1}$

$$
x\left(t_{1}\right)=A+B t_{1}+\int_{0}^{t_{1}} \int_{0}^{s} f(\sigma, x(\sigma)) d_{q_{0}} \sigma d_{q_{0}} s .
$$

For $t \in J_{1}=\left(t_{1}, t_{2}\right], q_{1}$-integrating (1.1), we have

$$
D_{q_{1}} x(t)=D_{q_{1}} x\left(t_{1}^{+}\right)+\int_{t_{1}}^{t} f(s, x(s)) d_{q_{1}} s
$$

Using the third condition of (1.1) with (3.3), it follows that

$$
D_{q_{1}} x(t)=B+\int_{0}^{t_{1}} f(s, x(s)) d_{q_{0}} s+I_{1}^{*}\left(x\left(t_{1}\right)\right)+\int_{t_{1}}^{t} f(s, x(s)) d_{q_{1}} s .
$$


Tariboon and Ntouyas Advances in Difference Equations 2014, 2014:31

Page 5 of 14

Taking the $q_{1}$-integral to (3.5) for $t \in J_{1}$, we obtain

$$
\begin{aligned}
x(t)= & x\left(t_{1}^{+}\right)+\left[B+\int_{0}^{t_{1}} f(s, x(s)) d_{q_{0}} s+I_{1}^{*}\left(x\left(t_{1}\right)\right)\right]\left(t-t_{1}\right) \\
& +\int_{t_{1}}^{t} \int_{t_{1}}^{s} f(\sigma, x(\sigma)) d_{q_{1}} \sigma d_{q_{1}} s .
\end{aligned}
$$

Applying the second equation of (1.1) with (3.4) and (3.6), we get

$$
\begin{aligned}
x(t)= & A+B t_{1}+\int_{0}^{t_{1}} \int_{0}^{s} f(\sigma, x(\sigma)) d_{q_{0}} \sigma d_{q_{0}} s+I_{1}\left(x\left(t_{1}\right)\right) \\
& +\left[B+\int_{0}^{t_{1}} f(s, x(s)) d_{q_{0}} s+I_{1}^{*}\left(x\left(t_{1}\right)\right)\right]\left(t-t_{1}\right) \\
& +\int_{t_{1}}^{t} \int_{t_{1}}^{s} f(\sigma, x(\sigma)) d_{q_{1}} \sigma d_{q_{1}} s \\
= & A+B t+\int_{0}^{t_{1}} \int_{0}^{s} f(\sigma, x(\sigma)) d_{q_{0}} \sigma d_{q_{0}} s+I_{1}\left(x\left(t_{1}\right)\right) \\
& +\left[\int_{0}^{t_{1}} f(s, x(s)) d_{q_{0}} s+I_{1}^{*}\left(x\left(t_{1}\right)\right)\right]\left(t-t_{1}\right) \\
& +\int_{t_{1}}^{t} \int_{t_{1}}^{s} f(\sigma, x(\sigma)) d_{q_{1}} \sigma d_{q_{1}} s .
\end{aligned}
$$

Repeating the above process, for $t \in J$, we get

$$
\begin{aligned}
x(t)= & A+B t \\
& +\sum_{0<t_{k}<t}\left(\int_{t_{k-1}}^{t_{k}} \int_{t_{k-1}}^{s} f(\sigma, x(\sigma)) d_{q_{k-1}} \sigma d_{q_{k-1}} s+I_{k}\left(x\left(t_{k}\right)\right)\right) \\
& +\sum_{0<t_{k}<t}\left(\int_{t_{k-1}}^{t_{k}} f(s, x(s)) d_{q_{k-1}} s+I_{k}^{*}\left(x\left(t_{k}\right)\right)\right)\left(t-t_{k}\right) \\
& +\int_{t_{k}}^{t} \int_{t_{k}}^{s} f(\sigma, x(\sigma)) d_{q_{k}} \sigma d_{q_{k}} s .
\end{aligned}
$$

The first boundary condition of (1.1) implies $A=0$. The second boundary condition of (1.1) yields

$$
\begin{aligned}
& \sum_{k=1}^{m}\left(\int_{t_{k-1}}^{t_{k}} \int_{t_{k-1}}^{s} f(\sigma, x(\sigma)) d_{q_{k-1}} \sigma d_{q_{k-1}} s+I_{k}\left(x\left(t_{k}\right)\right)\right) \\
& \quad+\sum_{k=1}^{m}\left(\int_{t_{k-1}}^{t_{k}} f(s, x(s)) d_{q_{k-1}} s+I_{k}^{*}\left(x\left(t_{k}\right)\right)\right)\left(T-t_{k}\right) \\
& \quad+\int_{t_{m}}^{T} \int_{t_{m}}^{s} f(\sigma, x(\sigma)) d_{q_{m}} \sigma d_{q_{m}} s+B T \\
& =\sum_{k=1}^{j}\left(\int_{t_{k-1}}^{t_{k}} \int_{t_{k-1}}^{s} f(\sigma, x(\sigma)) d_{q_{k-1}} \sigma d_{q_{k-1}} s+I_{k}\left(x\left(t_{k}\right)\right)\right)
\end{aligned}
$$




$$
\begin{aligned}
& +\sum_{k=1}^{j}\left(\int_{t_{k-1}}^{t_{k}} f(s, x(s)) d_{q_{k-1}} s+I_{k}^{*}\left(x\left(t_{k}\right)\right)\right)\left(\eta-t_{k}\right) \\
& +\int_{t_{j}}^{\eta} \int_{t_{j}}^{s} f(\sigma, x(\sigma)) d_{q_{j}} \sigma d_{q_{j}} s+B \eta
\end{aligned}
$$

which implies

$$
\begin{aligned}
B= & -\sum_{k=1}^{j}\left(\int_{t_{k-1}}^{t_{k}} f(s, x(s)) d_{q_{k-1}} s+I_{k}^{*}\left(x\left(t_{k}\right)\right)\right) \\
& -\frac{1}{T-\eta} \sum_{k=j+1}^{m}\left(\int_{t_{k-1}}^{t_{k}} \int_{t_{k-1}}^{s} f(\sigma, x(\sigma)) d_{q_{k-1}} \sigma d_{q_{k-1}} s+I_{k}\left(x\left(t_{k}\right)\right)\right) \\
& -\frac{1}{T-\eta} \sum_{k=j+1}^{m}\left(\int_{t_{k-1}}^{t_{k}} f(s, x(s)) d_{q_{k-1}} s+I_{k}^{*}\left(x\left(t_{k}\right)\right)\right)\left(T-t_{k}\right) \\
& +\frac{1}{T-\eta} \int_{t_{j}}^{\eta} \int_{t_{j}}^{s} f(\sigma, x(\sigma)) d_{q_{j}} \sigma d_{q_{j}} s-\frac{1}{T-\eta} \int_{t_{m}}^{T} \int_{t_{m}}^{s} f(\sigma, x(\sigma)) d_{q_{m}} \sigma d_{q_{m}} s .
\end{aligned}
$$

Substituting the constant $B$ into (3.7), we obtain (3.1) as required.

In view of Lemma 3.1, we define an operator $\mathcal{A}: P C(J, \mathbb{R}) \rightarrow P C(J, \mathbb{R})$ by

$$
\begin{aligned}
(\mathcal{A} x)(t)= & -t \sum_{k=1}^{j}\left(\int_{t_{k-1}}^{t_{k}} f(s, x(s)) d_{q_{k-1}} s+I_{k}^{*}\left(x\left(t_{k}\right)\right)\right) \\
& -\frac{t}{T-\eta} \sum_{k=j+1}^{m}\left(\int_{t_{k-1}}^{t_{k}} \int_{t_{k-1}}^{s} f(\sigma, x(\sigma)) d_{q_{k-1}} \sigma d_{q_{k-1}} s+I_{k}\left(x\left(t_{k}\right)\right)\right) \\
& -\frac{t}{T-\eta} \sum_{k=j+1}^{m}\left(\int_{t_{k-1}}^{t_{k}} f(s, x(s)) d_{q_{k-1}} s+I_{k}^{*}\left(x\left(t_{k}\right)\right)\right)\left(T-t_{k}\right) \\
& +\frac{t}{T-\eta} \int_{t_{j}}^{\eta} \int_{t_{j}}^{s} f(\sigma, x(\sigma)) d_{q_{j}} \sigma d_{q_{j}} s \\
& -\frac{t}{T-\eta} \int_{t_{m}}^{T} \int_{t_{m}}^{s} f(\sigma, x(\sigma)) d_{q_{m}} \sigma d_{q_{m}} s \\
& +\sum_{0<t_{k}<t}\left(\int_{t_{k-1}}^{t_{k}} \int_{t_{k-1}}^{s} f(\sigma, x(\sigma)) d_{q_{k-1}} \sigma d_{q_{k-1}} s+I_{k}\left(x\left(t_{k}\right)\right)\right) \\
& +\sum_{0<t_{k}<t}\left(\int_{t_{k-1}}^{t_{k}} f(s, x(s)) d_{q_{k-1}} s+I_{k}^{*}\left(x\left(t_{k}\right)\right)\right)\left(t-t_{k}\right) \\
& +\int_{t_{k}}^{t} \int_{t_{k}}^{s} f(\sigma, x(\sigma)) d_{q_{k}} \sigma d_{q_{k}} s . \\
&
\end{aligned}
$$

It should be noticed that problem (1.1) has solutions if and only if the operator $\mathcal{A}$ has fixed points. 
For convenience, we set

$$
\begin{aligned}
& \Phi_{k}=\left[\left(t_{k}-t_{k-1}\right)\left(T-t_{k}\right)+\frac{\left(t_{k}-t_{k-1}\right)^{2}}{1+q_{k-1}}\right] M_{1}+M_{2}+\left(T-t_{k}\right) M_{3}, \\
& \Psi_{k}=\left[\left(t_{k}-t_{k-1}\right)\left(T-t_{k}\right)+\frac{\left(t_{k}-t_{k-1}\right)^{2}}{1+q_{k-1}}\right] L_{1}+L_{2}+\left(T-t_{k}\right) L_{3},
\end{aligned}
$$

for $k=1, \ldots, m$.

\section{Theorem 3.2 Assume that:}

$\left(\mathrm{H}_{1}\right)$ The function $f:[0, T] \times \mathbb{R} \rightarrow \mathbb{R}$ is a continuous and there exists a constant $L_{1}>0$ such that $|f(t, x)-f(t, y)| \leq L_{1}|x-y|$, for each $t \in J$ and $x, y \in \mathbb{R}$.

$\left(\mathrm{H}_{2}\right)$ The functions $I_{k}, I_{k}^{*}: \mathbb{R} \rightarrow \mathbb{R}$ are continuous and there exist constants $L_{2}, L_{3}>0$ such that $\left|I_{k}(x)-I_{k}(y)\right| \leq L_{2}|x-y|$ and $\left|I_{k}^{*}(x)-I_{k}^{*}(y)\right| \leq L_{3}|x-y|$ for each $x, y \in \mathbb{R}, k=$ $1,2, \ldots, m$.

If

$$
\begin{aligned}
\Lambda:= & T \sum_{k=1}^{j}\left[\left(t_{k}-t_{k-1}\right) L_{1}+L_{3}\right]+\frac{T}{T-\eta} \sum_{k=j+1}^{m} \Psi_{k}+\frac{T L_{1}}{T-\eta}\left(\frac{\left(\eta-t_{j}\right)^{2}}{1+q_{j}}+\frac{\left(T-t_{m}\right)^{2}}{1+q_{m}}\right) \\
& +\sum_{k=1}^{m} \Psi_{k}+\frac{\left(T-t_{m}\right)^{2}}{1+q_{m}} L_{1} \leq \delta<1,
\end{aligned}
$$

then the impulsive $q_{k}$-difference boundary value problem (1.1) has a unique solution on J.

Proof First, we transform the problem (1.1) into a fixed-point problem, $x=\mathcal{A} x$, where the operator $\mathcal{A}$ is defined by (3.8). By using Banach's contraction principle, we shall show that $\mathcal{A}$ has a fixed point which is the unique solution of problem (1.1).

Set $\sup _{t \in J}|f(t, 0)|=M_{1}<\infty, \sup \left\{\left|I_{k}(0)\right|: k=1,2, \ldots, m\right\}=M_{2}<\infty, \sup \left\{\left|I_{k}^{*}(0)\right|: k=\right.$ $1,2, \ldots, m\}=M_{3}<\infty$ and a constant

$$
\begin{aligned}
\rho= & T \sum_{k=1}^{j}\left[\left(t_{k}-t_{k-1}\right) M_{1}+M_{3}\right]+\frac{T}{T-\eta} \sum_{k=j+1}^{m} \Phi_{k} \\
& +\frac{T M_{1}}{T-\eta}\left(\frac{\left(\eta-t_{j}\right)^{2}}{1+q_{j}}+\frac{\left(T-t_{m}\right)^{2}}{1+q_{m}}\right) \\
& +\sum_{k=1}^{m} \Phi_{k}+\frac{\left(T-t_{m}\right)^{2}}{1+q_{m}} M_{1} .
\end{aligned}
$$

Choosing $r \geq \frac{\rho}{1-\varepsilon}$, where $\delta \leq \varepsilon<1$, we show that $\mathcal{A} B_{r} \subset B_{r}$, where $B_{r}=\{x \in P C(J, \mathbb{R})$ : $\|x\| \leq r\}$. For $x \in B_{r}$, we have

$\|\mathcal{A} x\|$

$$
\leq \sup _{t \in J}\left\{t \sum_{k=1}^{j}\left(\int_{t_{k-1}}^{t_{k}}|f(s, x(s))| d_{q_{k-1}} s+\left|I_{k}^{*}\left(x\left(t_{k}\right)\right)\right|\right)\right.
$$


Tariboon and Ntouyas Advances in Difference Equations 2014, 2014:31

Page 8 of 14

http://www.advancesindifferenceequations.com/content/2014/1/31

$$
\begin{aligned}
& +\frac{t}{T-\eta} \sum_{k=j+1}^{m}\left(\int_{t_{k-1}}^{t_{k}} \int_{t_{k-1}}^{s}|f(\sigma, x(\sigma))| d_{q_{k-1}} \sigma d_{q_{k-1}} s+\left|I_{k}\left(x\left(t_{k}\right)\right)\right|\right) \\
& +\frac{t}{T-\eta} \sum_{k=j+1}^{m}\left(\int_{t_{k-1}}^{t_{k}}|f(s, x(s))| d_{q_{k-1}} s+\left|I_{k}^{*}\left(x\left(t_{k}\right)\right)\right|\right)\left(T-t_{k}\right) \\
& +\frac{t}{T-\eta} \int_{t_{j}}^{\eta} \int_{t_{j}}^{s}|f(\sigma, x(\sigma))| d_{q_{j}} \sigma d_{q_{j}} s+\frac{t}{T-\eta} \int_{t_{m}}^{T} \int_{t_{m}}^{s}|f(\sigma, x(\sigma))| d_{q_{m}} \sigma d_{q_{m}} s \\
& +\sum_{0<t_{k}<t}\left(\int_{t_{k-1}}^{t_{k}} \int_{t_{k-1}}^{s}|f(\sigma, x(\sigma))| d_{q_{k-1}} \sigma d_{q_{k-1}} s+\left|I_{k}\left(x\left(t_{k}\right)\right)\right|\right) \\
& +\sum_{0<t_{k}<t}\left(\int_{t_{k-1}}^{t_{k}}|f(s, x(s))| d_{q_{k-1}} s+\left|I_{k}^{*}\left(x\left(t_{k}\right)\right)\right|\right)\left(t-t_{k}\right) \\
& \left.+\int_{t_{k}}^{t} \int_{t_{k}}^{s}|f(\sigma, x(\sigma))| d_{q_{k}} \sigma d_{q_{k}} s\right\} \\
& \leq T \sum_{k=1}^{j}\left(\int_{t_{k-1}}^{t_{k}}(|f(s, x(s))-f(s, 0)|+|f(s, 0)|) d_{q_{k-1}} s+\left|I_{k}^{*}\left(x\left(t_{k}\right)\right)-I_{k}^{*}(0)\right|+\left|I_{k}^{*}(0)\right|\right) \\
& +\frac{T}{T-\eta} \sum_{k=j+1}^{m}\left(\int_{t_{k-1}}^{t_{k}} \int_{t_{k-1}}^{s}(|f(\sigma, x(\sigma))-f(\sigma, 0)|+|f(\sigma, 0)|) d_{q_{k-1}} \sigma d_{q_{k-1}} s\right. \\
& \left.+\left|I_{k}\left(x\left(t_{k}\right)\right)-I_{k}(0)\right|+\left|I_{k}(0)\right|\right) \\
& +\frac{T}{T-\eta} \sum_{k=j+1}^{m}\left(\int_{t_{k-1}}^{t_{k}}(|f(s, x(s))-f(s, 0)|+|f(s, 0)|) d_{q_{k-1}} s\right. \\
& \left.+\left|I_{k}^{*}\left(x\left(t_{k}\right)\right)-I_{k}^{*}(0)\right|+\left|I_{k}^{*}(0)\right|\right)\left(T-t_{k}\right) \\
& +\frac{T}{T-\eta} \int_{t_{j}}^{\eta} \int_{t_{j}}^{s}(|f(\sigma, x(\sigma))-f(\sigma, 0)|+|f(\sigma, 0)|) d_{q_{j}} \sigma d_{q_{j}} s \\
& +\frac{T}{T-\eta} \int_{t_{m}}^{T} \int_{t_{m}}^{s}(|f(\sigma, x(\sigma))-f(\sigma, 0)|+|f(\sigma, 0)|) d_{q_{m}} \sigma d_{q_{m}} s \\
& +\sum_{k=1}^{m}\left(\int_{t_{k-1}}^{t_{k}} \int_{t_{k-1}}^{s}(|f(\sigma, x(\sigma))-f(\sigma, 0)|+|f(\sigma, 0)|) d_{q_{k-1}} \sigma d_{q_{k-1}} s\right. \\
& \left.+\left|I_{k}\left(x\left(t_{k}\right)\right)-I_{k}(0)\right|+\left|I_{k}(0)\right|\right) \\
& +\sum_{k=1}^{m}\left(\int_{t_{k-1}}^{t_{k}}(|f(s, x(s))-f(s, 0)|+|f(s, 0)|) d_{q_{k-1}} s\right. \\
& \left.+\left|I_{k}^{*}\left(x\left(t_{k}\right)\right)-I_{k}^{*}(0)\right|+\left|I_{k}^{*}(0)\right|\right)\left(T-t_{k}\right) \\
& +\int_{t_{m}}^{T} \int_{t_{m}}^{s}(|f(\sigma, x(\sigma))-f(\sigma, 0)|+|f(\sigma, 0)|) d_{q_{m}} \sigma d_{q_{m}} s \\
& \leq T \sum_{k=1}^{j}\left(\left(t_{k}-t_{k-1}\right)\left(L_{1} r+M_{1}\right)+L_{3} r+M_{3}\right)
\end{aligned}
$$




$$
\begin{aligned}
& +\frac{T}{T-\eta} \sum_{k=j+1}^{m}\left(\frac{\left(t_{k}-t_{k-1}\right)^{2}}{1+q_{k-1}}\left(L_{1} r+M_{1}\right)+L_{2} r+M_{2}\right) \\
& +\frac{T}{T-\eta} \sum_{k=j+1}^{m}\left(\left(t_{k}-t_{k-1}\right)\left(L_{1} r+M_{1}\right)+L_{3} r+M_{3}\right)\left(T-t_{k}\right) \\
& +\frac{T}{T-\eta}\left(\frac{\left(\eta-t_{j}\right)^{2}}{1+q_{j}}+\frac{\left(T-t_{m}\right)^{2}}{1+q_{m}}\right)\left(L_{1} r+M_{1}\right) \\
& +\sum_{k=1}^{m}\left(\frac{\left(t_{k}-t_{k-1}\right)^{2}}{1+q_{k-1}}\left(L_{1} r+M_{1}\right)+L_{2} r+M_{2}\right) \\
& +\sum_{k=1}^{m}\left(\left(t_{k}-t_{k-1}\right)\left(L_{1} r+M_{1}\right)+L_{3} r+M_{3}\right)\left(T-t_{k}\right)+\frac{\left(T-t_{m}\right)^{2}}{1+q_{m}}\left(L_{1} r+M_{1}\right) \\
& =r \Lambda+\rho \leq(\delta+1-\varepsilon) r \leq r .
\end{aligned}
$$

It follows that $\mathcal{A} B_{r} \subset B_{r}$.

For $x, y \in P C(J, \mathbb{R})$ and for each $t \in J$, we have

$$
\begin{aligned}
\| \mathcal{A} x- & \mathcal{A} y \| \\
\leq & \sup _{t \in J}\left\{t \sum_{k=1}^{j}\left(\int_{t_{k-1}}^{t_{k}}|f(s, x(s))-f(s, y(s))| d_{q_{k-1}} s+\left|I_{k}^{*}\left(x\left(t_{k}\right)\right)-I_{k}^{*}\left(y\left(t_{k}\right)\right)\right|\right)\right. \\
& +\frac{t}{T-\eta} \sum_{k=j+1}^{m}\left(\int_{t_{k-1}}^{t_{k}} \int_{t_{k-1}}^{s}|f(\sigma, x(\sigma))-f(\sigma, y(\sigma))| d_{q_{k-1}} \sigma d_{q_{k-1}} s\right. \\
& \left.+\left|I_{k}\left(x\left(t_{k}\right)\right)-I_{k}\left(y\left(t_{k}\right)\right)\right|\right) \\
& +\frac{t}{T-\eta} \sum_{k=j+1}^{m}\left(\int_{t_{k-1}}^{t_{k}}|f(s, x(s))-f(s, y(s))| d_{q_{k-1}} s+\left|I_{k}^{*}\left(x\left(t_{k}\right)\right)-I_{k}^{*}\left(y\left(t_{k}\right)\right)\right|\right)\left(T-t_{k}\right) \\
& +\frac{t}{T-\eta} \int_{t_{j}}^{\eta} \int_{t_{j}}^{s}|f(\sigma, x(\sigma))-f(\sigma, y(\sigma))| d_{q_{j}} \sigma d_{q_{j}} s \\
& +\frac{t}{T-\eta} \int_{t_{m}}^{T} \int_{t_{m}}^{s}|f(\sigma, x(\sigma))-f(\sigma, y(\sigma))| d_{q_{m}} \sigma d_{q_{m}} s \\
& +\sum_{0<t_{k}<t}\left(\int_{t_{k-1}}^{t_{k}} \int_{t_{k-1}}^{s}|f(\sigma, x(\sigma))-f(\sigma, y(\sigma))| d_{q_{k-1}} \sigma d_{q_{k-1}} s+\left|I_{k}\left(x\left(t_{k}\right)\right)-I_{k}\left(y\left(t_{k}\right)\right)\right|\right) \\
& +\sum_{0<t_{k}<t}\left(\int_{t_{k-1}}^{t_{k}}|f(s, x(s))-f(s, y(s))| d_{q_{k-1}} s+\left|I_{k}^{*}\left(x\left(t_{k}\right)\right)-I_{k}^{*}\left(y\left(t_{k}\right)\right)\right|\right)\left(t-t_{k}\right) \\
& \left.+\int_{t_{k}}^{t} \int_{t_{k}}^{s}|f(\sigma, x(\sigma))-f(\sigma, y(\sigma))| d_{q_{k}} \sigma d_{q_{k}} s\right\} \\
& +\frac{T\|x-y\|}{T-\eta} \sum_{k=j+1}^{m}\left(\left(t_{k}-t_{k-1}\right) L_{1}+L_{3}\right)\left(T-t_{k}\right) \\
&
\end{aligned}
$$




$$
\begin{aligned}
& +\frac{T\|x-y\|}{T-\eta}\left(\frac{\left(\eta-t_{j}\right)^{2}}{1+q_{j}}+\frac{\left(T-t_{m}\right)^{2}}{1+q_{m}}\right) L_{1} \\
& +\sum_{k=1}^{m}\left(\frac{\left(t_{k}-t_{k-1}\right)^{2}}{1+q_{k-1}} L_{1}+L_{2}\right)\|x-y\|+\sum_{k=1}^{m}\left(\left(t_{k}-t_{k-1}\right) L_{1}+L_{3}\right)\left(T-t_{k}\right)\|x-y\| \\
& +\frac{\left(T-t_{m}\right)^{2}}{1+q_{m}} L_{1}\|x-y\| \\
& =\Lambda\|x-y\| .
\end{aligned}
$$

As $\Lambda<1, \mathcal{A}$ is a contraction. Hence, by Banach's contraction mapping principle, we find that $\mathcal{A}$ has a fixed point which is the unique solution of problem (1.1).

Our next result is based on Krasnoselskii's fixed-point theorem.

Lemma 3.3 (Krasnoselskii's fixed-point theorem) [19] Let $M$ be a closed, bounded, convex and nonempty subset of a Banach space X. Let A, B be the operators such that (a) Ax+By $\in$ $M$ whenever $x, y \in M$; (b) $A$ is compact and continuous; (c) $B$ is a contraction mapping. Then there exists $z \in M$ such that $z=A z+B z$.

Further, we use the notation

$$
\begin{aligned}
\theta_{1}= & T \sum_{k=1}^{j}\left(t_{k}-t_{k-1}\right)+\frac{T}{T-\eta} \sum_{k=j+1}^{m} \frac{\left(t_{k}-t_{k-1}\right)^{2}}{1+q_{k-1}} \\
& +\frac{T}{T-\eta} \sum_{k=j+1}^{m}\left(T-t_{k}\right)\left(t_{k}-t_{k-1}\right)+\frac{T\left(\eta-t_{j}\right)^{2}}{(T-\eta)\left(1+q_{j}\right)} \\
& +\frac{T\left(T-t_{m}\right)^{2}}{(T-\eta)\left(1+q_{m}\right)}+\sum_{k=1}^{m+1} \frac{\left(t_{k}-t_{k-1}\right)^{2}}{1+q_{k-1}}+\sum_{k=1}^{m}\left(T-t_{k}\right)\left(t_{k}-t_{k-1}\right),
\end{aligned}
$$

and

$$
\theta_{2}=j T N_{2}+\frac{(m-j) T N_{1}}{T-\eta}+m N_{1}+N_{2} \sum_{k=1}^{m}\left(T-t_{k}\right)+\frac{T N_{2}}{T-\eta} \sum_{j+1}^{m}\left(T-t_{k}\right) .
$$

Theorem 3.4 Let $f: J \times \mathbb{R} \rightarrow \mathbb{R}$ be a continuous function. Assume that $\left(\mathrm{H}_{2}\right)$ holds and in addition suppose that:

$\left(\mathrm{H}_{3}\right)|f(t, x)| \leq \mu(t), \forall(t, x) \in J \times \mathbb{R}$, and $\mu \in C\left(J, \mathbb{R}^{+}\right)$.

$\left(\mathrm{H}_{4}\right)$ There exist constants $N_{1}, N_{2}>0$ such that $\left|I_{k}(x)\right| \leq N_{1}$ and $\left|I_{k}^{*}(x)\right| \leq N_{2}$ for all $x \in \mathbb{R}$, for $k=1,2, \ldots, m$.

Then the impulsive $q_{k}$-difference boundary value problem (1.1) has at least one solution on I provided that

$$
j T L_{3}+m L_{2}+\frac{T(m-j) L_{2}}{T-\eta}+L_{3} \sum_{k=1}^{m}\left(T-t_{k}\right)<1 .
$$


Proof Firstly, we define $\sup _{t \in J}|\mu(t)|=\|\mu\|$. Choosing a suitable ball $B_{R}=\{x \in P C(J, \mathbb{R})$ : $\|x\| \leq R\}$, where

$$
R \geq\|\mu\| \theta_{1}+\theta_{2}
$$

and $\theta_{1}, \theta_{2}$ are defined by (3.13), (3.14), respectively, we define the operators $\mathcal{S}_{1}$ and $\mathcal{S}_{2}$ on $B_{R}$ by

$\left(\mathcal{S}_{1} x\right)(t)$

$$
\begin{aligned}
= & -t \sum_{k=1}^{j} \int_{t_{k-1}}^{t_{k}} f(s, x(s)) d_{q_{k-1}} s-\frac{t}{T-\eta} \sum_{k=j+1}^{m} \int_{t_{k-1}}^{t_{k}} \int_{t_{k-1}}^{s} f(\sigma, x(\sigma)) d_{q_{k-1}} \sigma d_{q_{k-1}} s \\
& -\frac{t}{T-\eta} \sum_{k=j+1}^{m}\left(T-t_{k}\right) \int_{t_{k-1}}^{t_{k}} f(s, x(s)) d_{q_{k-1}} s+\frac{t}{T-\eta} \int_{t_{j}}^{\eta} \int_{t_{j}}^{s} f(\sigma, x(\sigma)) d_{q_{j}} \sigma d_{q_{j}} s \\
& -\frac{t}{T-\eta} \int_{t_{m}}^{T} \int_{t_{m}}^{s} f(\sigma, x(\sigma)) d_{q_{m}} \sigma d_{q_{m}} s+\sum_{0<t_{k}<t} \int_{t_{k-1}}^{t_{k}} \int_{t_{k-1}}^{s} f(\sigma, x(\sigma)) d_{q_{k-1}} \sigma d_{q_{k-1}} s \\
& +\sum_{0<t_{k}<t}\left(t-t_{k}\right) \int_{t_{k-1}}^{t_{k}} f(s, x(s)) d_{q_{k-1}} s+\int_{t_{k}}^{t} \int_{t_{k}}^{s} f(\sigma, x(\sigma)) d_{q_{k}} \sigma d_{q_{k}} s, \quad t \in[0, T],
\end{aligned}
$$

and

$$
\begin{aligned}
\left(\mathcal{S}_{2} x\right)(t)= & -t \sum_{k=1}^{j} I_{k}^{*}\left(x\left(t_{k}\right)\right)-\frac{t}{T-\eta} \sum_{k=j+1}^{m} I_{k}\left(x\left(t_{k}\right)\right)-\frac{t}{T-\eta} \sum_{k=j+1}^{m}\left(T-t_{k}\right) I_{k}^{*}\left(x\left(t_{k}\right)\right) \\
& +\sum_{0<t_{k}<t} I_{k}\left(x\left(t_{k}\right)\right)+\sum_{0<t_{k}<t}\left(t-t_{k}\right) I_{k}^{*}\left(x\left(t_{k}\right)\right), \quad t \in[0, T] .
\end{aligned}
$$

For any $x, y \in B_{R}$, we have

$$
\begin{aligned}
\left\|\mathcal{S}_{1} x+\mathcal{S}_{2} y\right\| \leq & \|\mu\|\left[T \sum_{k=1}^{j}\left(t_{k}-t_{k-1}\right)+\frac{T}{T-\eta} \sum_{k=j+1}^{m} \frac{\left(t_{k}-t_{k-1}\right)^{2}}{1+q_{k-1}}\right. \\
& +\frac{T}{T-\eta} \sum_{k=j+1}^{m}\left(T-t_{k}\right)\left(t_{k}-t_{k-1}\right)+\frac{T\left(\eta-t_{j}\right)^{2}}{(T-\eta)\left(1+q_{j}\right)} \\
& \left.+\frac{T\left(T-t_{m}\right)^{2}}{(T-\eta)\left(1+q_{m}\right)}+\sum_{k=1}^{m+1} \frac{\left(t_{k}-t_{k-1}\right)^{2}}{1+q_{k-1}}+\sum_{k=1}^{m}\left(T-t_{k}\right)\left(t_{k}-t_{k-1}\right)\right] \\
& +j T N_{2}+\frac{(m-j) T N_{1}}{T-\eta}+m N_{1}+N_{2} \sum_{k=1}^{m}\left(T-t_{k}\right)+\frac{T N_{2}}{T-\eta} \sum_{j+1}^{m}\left(T-t_{k}\right) \\
= & \|\mu\| \theta_{1}+\theta_{2} \\
\leq & R .
\end{aligned}
$$

Hence, $\mathcal{S}_{1} x+\mathcal{S}_{2} y \in B_{R}$. 
To show that $\mathcal{S}_{2}$ is a contraction, for $x, y \in P C(J, \mathbb{R})$, we have

$$
\begin{aligned}
\left\|\mathcal{S}_{2} x-\mathcal{S}_{2} y\right\| \leq & T \sum_{k=1}^{j}\left|I_{k}^{*}\left(x\left(t_{k}\right)\right)-I_{k}^{*}\left(y\left(t_{k}\right)\right)\right|+\frac{T}{T-\eta} \sum_{k=j+1}^{m}\left|I_{k}\left(x\left(t_{k}\right)\right)-I_{k}\left(y\left(t_{k}\right)\right)\right| \\
& +\sum_{k=1}^{m}\left|I\left(x\left(t_{k}\right)\right)-I_{k}\left(y\left(t_{k}\right)\right)\right|+\sum_{k=1}^{m}\left(t-t_{k}\right)\left|I_{k}^{*}\left(x\left(t_{k}\right)\right)-I_{k}^{*}\left(y\left(t_{k}\right)\right)\right| \\
\leq & {\left[j T L_{3}+m L_{2}+\frac{T(m-j) L_{2}}{T-\eta}+L_{3} \sum_{k=1}^{m}\left(T-t_{k}\right)\right]\|x-y\| . }
\end{aligned}
$$

From (3.15), it follows that $\mathcal{S}_{2}$ is a contraction.

Next, the continuity of $f$ implies that the operator $\mathcal{S}_{1}$ is continuous. Further, $\mathcal{S}_{1}$ is uniformly bounded on $B_{R}$ by

$\left\|\mathcal{S}_{1} x\right\| \leq\|\mu\| \theta_{1}$.

Now we shall prove the compactness of $\mathcal{S}_{1}$. Setting $\sup _{(t, x) \in J \times B_{R}}|f(t, x)|=f^{*}<\infty$, then for each $\tau_{1}, \tau_{2} \in\left(t_{l}, t_{l+1}\right)$ for some $l \in\{0,1, \ldots, m\}$ with $\tau_{2}>\tau_{1}$, we have

$$
\begin{aligned}
\mid\left(\mathcal{S}_{1} x\right) & \left(\tau_{2}\right)-\left(\mathcal{S}_{1} x\right)\left(\tau_{1}\right) \mid \\
\leq & \left|\tau_{2}-\tau_{1}\right| \sum_{k=1}^{j} \int_{t_{k-1}}^{t_{k}}|f(s, x(s))| d_{q_{k-1}} s \\
& +\frac{\left|\tau_{2}-\tau_{1}\right|}{T-\eta} \sum_{k=j+1}^{m} \int_{t_{k-1}}^{t_{k}} \int_{t_{k-1}}^{s}|f(\sigma, x(\sigma))| d_{q_{k-1}} \sigma d_{q_{k-1}} s \\
& +\frac{\left|\tau_{2}-\tau_{1}\right|}{T-\eta} \sum_{k=j+1}^{m}\left(T-t_{k}\right) \int_{t_{k-1}}^{t_{k}}|f(s, x(s))| d_{q_{k-1}} s \\
& +\frac{\left|\tau_{2}-\tau_{1}\right|}{T-\eta} \int_{t_{j}}^{\eta} \int_{t_{j}}^{s}|f(\sigma, x(\sigma))| d_{q_{j}} \sigma d_{q_{j}} s \\
& +\frac{\left|\tau_{2}-\tau_{1}\right|}{T-\eta} \int_{t_{m}}^{T} \int_{t_{m}}^{s}|f(\sigma, x(\sigma))| d_{q_{m}} \sigma d_{q_{m}} s+\left|\tau_{2}-\tau_{1}\right| \sum_{k=1}^{l} \int_{t_{k-1}}^{t_{k}}|f(s, x(s))| d_{q_{k-1}} s \\
& +\left|\int_{t_{l}}^{\tau_{2}} \int_{t_{l}}^{s}\right| f(\sigma, x(\sigma))\left|d_{q_{l}} \sigma d_{q_{l}} s-\int_{t_{l}}^{\tau_{1}} \int_{t_{l}}^{s}\right| f(\sigma, x(\sigma))\left|d_{q_{l}} \sigma d_{q_{l}} s\right| \\
\leq & \left|\tau_{2}-\tau_{1}\right| f^{*}\left[\sum_{k=1}^{j}\left(t_{k}-t_{k-1}\right)+\frac{1}{T-\eta} \sum_{k=j+1}^{m} \frac{\left(t_{k}-t_{k-1}\right)^{2}}{1+q_{k-1}}+\frac{\left(\eta-t_{j}\right)^{2}}{(T-\eta)\left(1+q_{j}\right)}\right. \\
& +\frac{\left(T-t_{m}\right)^{2}}{(T-\eta)\left(1+q_{m}\right)}+\frac{1}{T-\eta} \sum_{k=j+1}^{m}\left(T-t_{k}\right)\left(t_{k}-t_{k-1}\right) \\
& \left.+\sum_{k=1}^{l}\left(t_{k}-t_{k-1}\right)+\frac{\left(\tau_{1}+\tau_{2}+2 t_{l}\right)}{1+q_{l}}\right] . \\
&
\end{aligned}
$$

As $\tau_{1} \rightarrow \tau_{2}$, the right hand side above (which is independent of $x$ ) tends to zero. Therefore, the operator $\mathcal{S}_{1}$ is equicontinuous. Since $\mathcal{S}_{1}$ maps bounded subsets into relatively compact 
subsets, it follows that $\mathcal{S}_{1}$ is relative compact on $B_{R}$. Hence, by the Arzelá-Ascoli theorem, $\mathcal{S}_{1}$ is compact on $B_{R}$. Thus all the assumptions of Lemma 3.3 are satisfied. Hence, by the conclusion of Lemma 3.3, the impulsive $q_{k}$-difference boundary value problem (1.1) has at least one solution on $J$.

\section{Examples}

Example 4.1 Consider the following nonlinear second-order impulsive $q_{k}$-difference equation with three-point boundary condition:

$$
\left\{\begin{array}{l}
D_{\frac{4}{5+k}}^{2} x(t)=\frac{e^{-\cos ^{2} t}|x(t)|}{(6+t)^{2}(1+|x(t)|)}, \quad t \in J=[0,1], t \neq t_{k}=\frac{k}{10}, \\
\Delta x\left(t_{k}\right)=\frac{\left|x\left(t_{k}\right)\right|}{8\left(7+\left|x\left(t_{k}\right)\right|\right)}, \quad k=1,2, \ldots, 9, \\
D_{\frac{4}{5+k}} x\left(t_{k}^{+}\right)-D_{\frac{4}{4+k}} x\left(t_{k}\right)=\frac{1}{6} \tan ^{-1}\left(\frac{1}{8} x\left(t_{k}\right)\right), \quad k=1,2, \ldots, 9, \\
x(0)=0, \quad x(1)=x\left(\frac{1}{4}\right) .
\end{array}\right.
$$

Here $q_{k}=4 /(5+k)$ for $k=0,1,2, \ldots, 9, m=9, T=1, \eta=1 / 4, j=2, f(t, x)=\left(e^{-\cos ^{2} t}|x|\right) /$ $\left((6+t)^{2}(1+|x|)\right), I_{k}(x)=|x| /(8(7+|x|))$ and $I_{k}^{*}(x)=(1 / 6) \tan ^{-1}(x / 8)$. Since

$$
\begin{aligned}
& |f(t, x)-f(t, y)| \leq(1 / 36)|x-y|, \\
& \left|I_{k}(x)-I_{k}(y)\right| \leq(1 / 56)|x-y| \quad \text { and } \quad\left|I_{k}^{*}(x)-I_{k}^{*}(y)\right| \leq(1 / 48)|x-y|,
\end{aligned}
$$

then $\left(\mathrm{H}_{1}\right)$ and $\left(\mathrm{H}_{2}\right)$ are satisfied with $L_{1}=(1 / 36), L_{2}=(1 / 56), L_{3}=(1 / 48)$. We can show that

$$
\Lambda \approx 0.5730986482<1
$$

Hence, by Theorem 3.2, the three-point impulsive $q_{k}$-difference boundary value problem (4.1) has a unique solution on $[0,1]$.

Example 4.2 Consider the following nonlinear second-order impulsive $q_{k}$-difference equation with three-point boundary condition:

$$
\left\{\begin{array}{l}
D_{\frac{3}{6+k}}^{2} x(t)=\frac{\sin ^{2}(\pi t)}{(t+4)^{2}} \frac{|x(t)|}{(1+|x(t)|)}, \quad t \in J=[0,1], t \neq t_{k}=\frac{k}{10}, \\
\Delta x\left(t_{k}\right)=\frac{\left|x\left(t_{k}\right)\right|}{9\left(7+\left|x\left(t_{k}\right)\right|\right)}, \quad k=1,2, \ldots, 9, \\
D_{\frac{3}{6+k}} x\left(t_{k}^{+}\right)-D_{\frac{3}{5+k}} x\left(t_{k}\right)=\frac{\left|x\left(t_{k}\right)\right|}{4\left(5+\left|x\left(t_{k}\right)\right|\right)}, \quad k=1,2, \ldots, 9 \\
x(0)=0, \quad x(1)=x\left(\frac{9}{20}\right) .
\end{array}\right.
$$

Set $q_{k}=3 /(6+k)$ for $k=0,1,2, \ldots, 9, m=9, T=1, \eta=9 / 20, j=4, f(t, x)=\left(\sin ^{2}(\pi t)|x|\right) /$ $\left((t+4)^{2}(1+|x|)\right), I_{k}(x)=|x| /(9(7+|x|))$ and $I_{k}^{*}(x)=|x| /(4(5+|x|))$. Since

$$
\left|I_{k}(x)-I_{k}(y)\right| \leq(1 / 63)|x-y| \quad \text { and } \quad\left|I_{k}^{*}(x)-I_{k}^{*}(y)\right| \leq(1 / 20)|x-y| \text {, }
$$

then $\left(\mathrm{H}_{2}\right)$ is satisfied with $L_{2}=(1 / 63), L_{3}=(1 / 20)$. It is easy to verify that $|f(t, x)| \leq \mu(t) \equiv 1$, $I_{k}(x) \leq N_{1}=1 / 9$ and $I_{k}^{*}(x) \leq N_{2}=1 / 4$ for all $t \in[0,1], x \in \mathbb{R}, k=1, \ldots, m$. Thus $\left(\mathrm{H}_{3}\right)$ and $\left(\mathrm{H}_{4}\right)$ are satisfied. We can show that

$$
j T L_{3}+m L_{2}+\frac{T(m-j) L_{2}}{T-\eta}+L_{3} \sum_{k=1}^{m}\left(T-t_{k}\right)=\frac{19741}{27720}<1 .
$$


Hence, by Theorem 3.3, the three-point impulsive $q_{k}$-difference boundary value problem (4.2) has at least one solution on $[0,1]$.

\section{Competing interests}

The authors declare that they have no competing interests.

\section{Authors' contributions}

Both authors contributed equally in this article. They read and approved the final manuscript.

\section{Author details}

'Department of Mathematics, Faculty of Applied Science, King Mongkut's University of Technology North Bangkok, Bangkok, Thailand. ${ }^{2}$ Department of Mathematics, University of loannina, loannina, 451 10, Greece.

\section{Authors' information}

Sotiris K Ntouyas is a member of Nonlinear Analysis and Applied Mathematics (NAAM)-Research Group at King Abdulaziz University, Jeddah, Saudi Arabia.

\section{Acknowledgements}

This research of J Tariboon is supported by King Mongkut's University of Technology North Bangkok, Thailand.

Received: 11 November 2013 Accepted: 7 January 2014 Published: 27 Jan 2014

\section{References}

1. Tariboon, J, Ntouyas, SK: Quantum calculus on finite intervals and applications to impulsive difference equations. Adv. Differ. Equ. 2013, 282 (2013)

2. Kac, V, Cheung, P: Quantum Calculus. Springer, New York (2002)

3. Bangerezako, G: Variational q-calculus. J. Math. Anal. Appl. 289, 650-665 (2004)

4. Dobrogowska, A, Odzijewicz, A: Second order q-difference equations solvable by factorization method. J. Comput. Appl. Math. 193, 319-346 (2006)

5. Gasper, G, Rahman, M: Some systems of multivariable orthogonal q-Racah polynomials. Ramanujan J. 13, $389-405$ (2007)

6. Ismail, MEH, Simeonov, P: q-Difference operators for orthogonal polynomials. J. Comput. Appl. Math. 233, 749-761 (2009)

7. Bohner, M, Guseinov, GS: The $h$-Laplace and q-Laplace transforms. J. Math. Anal. Appl. 365, 75-92 (2010)

8. El-Shahed, M, Hassan, HA: Positive solutions of q-difference equation. Proc. Am. Math. Soc. 138, 1733-1738 (2010)

9. Ahmad, B: Boundary-value problems for nonlinear third-order q-difference equations. Electron. J. Differ. Equ. 94, 1-7 (2011)

10. Ahmad, B, Alsaedi, A, Ntouyas, SK: A study of second-order q-difference equations with boundary conditions. Adv. Differ. Equ. 2012, 35 (2012)

11. Ahmad, B, Ntouyas, SK, Purnaras, IK: Existence results for nonlinear q-difference equations with nonlocal boundary conditions. Commun. Appl. Nonlinear Anal. 19, 59-72 (2012)

12. Ahmad, B, Nieto, JJ: On nonlocal boundary value problems of nonlinear q-difference equations. Adv. Differ. Equ. 2012, 81 (2012)

13. Ahmad, B, Ntouyas, SK: Boundary value problems for q-difference inclusions. Abstr. Appl. Anal. 2011, Article ID 292860 (2011)

14. Zhou, W, Liu, H: Existence solutions for boundary value problem of nonlinear fractional q-difference equations. Adv. Differ. Equ. 2013, 113 (2013)

15. Yu, C, Wang, J: Existence of solutions for nonlinear second-order q-difference equations with first-order q-derivatives. Adv. Differ. Equ. 2013, 124 (2013)

16. Lakshmikantham, V, Bainov, DD, Simeonov, PS: Theory of Impulsive Differential Equations. World Scientific, Singapore (1989)

17. Samoilenko, AM, Perestyuk, NA: Impulsive Differential Equations. World Scientific, Singapore (1995)

18. Benchohra, M, Henderson, J, Ntouyas, SK: Impulsive Differential Equations and Inclusions, vol. 2. Hindawi Publishing Corporation, New York (2006)

19. Krasnoselskii, MA: Two remarks on the method of successive approximations. Usp. Mat. Nauk 10, $123-127$ (1955)

10.1186/1687-1847-2014-31

Cite this article as: Tariboon and Ntouyas: Three-point boundary value problems for nonlinear second-order impulsive q-difference equations. Advances in Difference Equations 2014, 2014:31 